\title{
Analysis of the NANDA-I taxonomy "maternal-fetal dyad" concept in high-risk pregnancy: integrative review
}

\author{
Análise do conceito "binômio mãe-feto" da taxonomia da NANDA-I em gestantes de alto risco: revisão integrativa \\ Análisis del concepto "binomio madre-feto" de la taxonomía nanda-i en mujeres embarazadas de alto riesgo: \\ revisión integrativa
} Ryanne Carolynne Marques Gomes'
ORCID: 0000-0001-7554-2662

Marcos Venícios de Oliveira Lopes" ORCID: 0000-0001-5867-8023

Jaqueline Galdino Albuquerque Perrelli' ORCID: 0000-0003-4934-1335

Cleide Maria Pontes' ORCID: 0000-0003-4707-6873

Francisca Márcia Pereira Linhares' ORCID: 0000-0001-9778-5024

Suzana de Oliveira Mangueira' ORCID: 0000-0003-0931-8675

'Universidade Federal de Pernambuco. Recife, Pernambuco, Brazil. "Universidade Federal do Ceará. Fortaleza, Ceará, Brazil.

How to cite this article: Gomes RCM, Lopes MVO, Perrelli JGA, Pontes CM, Linhares FMP, Mangueira SO. Analysis of the NANDA-I taxonomy "maternal-fetal dyad" concept in high-risk pregnancy: integrative review. Rev Bras Enferm. 2020;73(Suppl 4):e20190649. doi: http://dx.doi.org/10.1590/0034-7167-2019-0649

\section{Corresponding author:}

Ryanne Carolynne Marques Gomes E-mail: ryannecarolynne@gmail.com

EDITOR IN CHIEF: Antonio José de Almeida Filho ASSOCIATE EDITOR: Hugo Fernandes

\section{ABSTRACT}

Objective: to analyze the NANDA-I taxonomy "maternal-fetal dyad" concept in high-risk pregnancy. Method: an integrative literature review based on the Conceptual Analysis model proposed by Walker and Avant. It was conducted at PUBMED, CINAHL, SCOPUS, LILACS and SciELO, with the descriptors: Complications of pregnancy, Risk Factors, and Pregnancy, High-Risk (from 2008-2020). Result: the sample consisted of 44 articles, which identified two attributes and 21 antecedents of the concept. Conclusion: concept analysis allowed to identify the attributes and antecedents of the "maternal-fetal dyad" concept in high-risk pregnancy, in addition to raising and clarifying ideas. It was possible to identify 12 antecedents that are not included in NANDA-I as well as the need to review the definition of the diagnosis proposed by the taxonomy.

Descriptors: Nursing Diagnosis; Risk Factors; Fetus; High Risk Pregnancy; Review.

\section{RESUMO}

Objetivo: analisar o conceito "binômio mãe-feto" da taxonomia da NANDA-I em gestantes de alto risco. Método: revisão integrativa da literatura, fundamentada no modelo de Análise Conceitual proposto por Walker e Avant. Realizada nas bases de dados PubMed, CINAHL, Scopus, LILACS e na biblioteca eletrônica SciELO, com os descritores: Pregnancy Complications, Risk Factors e Pregnancy, High-Risk, com recorte temporal de 2008-2020. Resultado: a amostra constituiu-se de 44 artigos, que identificaram dois atributos e 21 antecedentes do conceito. Conclusão: a análise de conceito permitiu identificar os atributos e os antecedentes do conceito "binômio mãe-feto" em gestantes de alto risco, além de suscitar e esclarecer ideias. Foi possível identificar 12 antecedentes que não constam na NANDA-I, bem como a necessidade de revisar a definição do diagnóstico proposta pela taxonomia.

Descritores: Diagnóstico de Enfermagem; Fatores de Risco; Feto; Gravidez de Alto Risco; Revisão.

\section{RESUMEN}

Objetivo: analizar el concepto "binomio madre-feto"'"' de la taxonomía NANDA-I en mujeres embarazadas de alto riesgo. Método: una revisión bibliográfica integradora, basada en el modelo de Análisis Conceptual propuesto por Walker y Avant. Realizado en las bases de datos PubMed, CINAHL, Scopus, LILACS y en la biblioteca electrónica SciELO, con los descriptores: Pregnancy Complications, Risk Factors y Pregnancy, High-Risk, con un marco temporal de 2008-2020. Resultado: la muestra consistió en 44 artículos, que identificaron dos atributos y 21 antecedentes del concepto. Conclusión: el análisis conceptual permitió identificar los atributos y antecedentes del concepto "binomio madre-feto" en mujeres embarazadas de alto riesgo, planteando y aclarando ideas. Fue posible identificar 12 antecedentes que no están incluidos en NANDA-I, así como la necesidad de revisar la definición del diagnóstico propuesto por la taxonomía.

Descriptores: Diagnóstico de Enfermería; Factores de Riesgo; Feto; Embarazo de Alto Riesgo; Revisión. 


\section{INTRODUCTION}

Threat to the well-being of the maternal-fetal dyad in the gestational period arises from several clinical, obstetric, and social conditions that can cause maternal and/or fetal complications and compromise their outcome. Pregnancy that occurs with complications corresponds to $20 \%$ of the total pregnancies and is called high risk ${ }^{(1)}$.

Due to the unexpected conditions associated with pregnan$c y$, there is a real or potential risk to the health and well-being of the mother and/or the child. Risk assessment is necessary to identify any condition that may impact the dyad. Assistance to pregnant women, with detailed antecedents, complete physical examination and laboratory findings may reveal risks of maternal and/or fetal mortality or morbidity ${ }^{(2)}$.

In assisting high-risk pregnancy, nurses must provide proper care and act in promoting the health of women and the child ${ }^{(1-2)}$. The methodological instrument used to guide care actions is the Nursing Process (NP), which supports the Systematization of Nursing Care (SNC). NP assists nurses in noticing individuals' health problems, planning and implementing their actions, as well as assessing the results ${ }^{(3)}$. NP consists of data collection, nursing diagnosis, planning, implementation, and assessment ${ }^{(4)}$.

To promote care, we seek to implement the $\mathrm{SNC}^{(3)}$. In order to carry out its accomplishment, some nursing classifications were developed to standardize the language among nurses. Among them is the NANDA International (NANDA-I) taxonomy ${ }^{(4)}$, which allows nurses to identify nursing diagnoses (ND) according to the needs of the individual, family, or community.

In 2008, NANDA-I integrated the "risk for disturbed maternal-fetal dyad" ND, which is inserted in the "sexuality" domain and in the "reproduction" class. Diagnosis is defined by NANDA-I as "at risk for disruption of the symbiotic maternal-fetal dyad as a result of comorbidity or pregnancy-related conditions"(4). Its level of evidence is 2.1, which corresponds to the ND that was accepted for integration and publication in NANDA-I, since literature was cited both for its definition and for its clinical indicators ${ }^{(4)}$. However, no integrative literature review or conceptual analysis studies have been carried out that depict evidence that supports the components of the diagnosis and so that its level of evidence can increase.

The $11^{\text {th }}$ edition of NANDA-I (2018-2020) ${ }^{(4)}$ recategorized some risk factors in populations at risk (group of people who have characteristics that are susceptible to a specific human response that cannot be modified by nurses independently) and in related conditions (diagnoses) injuries, procedures, medical devices or pharmaceutical agents, which are not independently modifiable by nurses). The diagnosis under study consists of three risk factors, four related conditions and no population at risk. Thus, it is necessary to review the elements of the "risk for disturbed maternal-fetal dyad" ND in high-risk pregnancy.

This research contributes to improve the maternal-fetal dyad concept, which is relevant to understand the symbiotic relationship between the mother and the child, and to guide the implementation of SNC. Moreover, it offers subsidies for nurses' clinical practice in assisting pregnant women, especially at high-risk. Concept analysis will be the basis to proceed with the development of validation of the ND under study.

\section{OBJECTIVE}

To analyze the NANDA-I taxonomy "maternal-fetal dyad" concept in high-risk pregnancy.

\section{METHODS}

\section{Type of study}

This is the concept analysis, which was carried out through the theoretical framework of Walker and Avant ${ }^{(5)}$ and through an integrative review, concomitantly.

The concept analysis corresponds to the first stage of ND validation studies and consists of eight stages, proposed by Walker and Avant ${ }^{(5)}: 1$. Choosing a concept; 2. Determining the purpose of analysis; 3 . Identifying all uses of the concept; 4. Defining attributes; 5 . Identifying a model case; 6 . Identifying borderline, related, and contrary cases; 7 . Identifying antecedents and consequences; 8. Defining empirical references.

For this study, we carried out six steps: choosing a concept, determining the purpose of analysis, defining attributes, identifying antecedents and consequences (risk ND contains only antecedents - risk factors, population at risk, and related conditions) ${ }^{(4)}$, identifying a model case, and identifying borderline, related, and contrary cases. Next, the steps performed and their operation will be described:

1. Choosing a concept: the chosen concept was the "maternal-fetal dyad" of NANDA-I taxonomy. This concept emerged from the conceptual core of the "risk for disturbed maternal-fetal dyad" ND.

2. Determining the purpose of analysis: this analysis aimed to identify the critical attributes and antecedents of the NANDA-I taxonomy "maternal-fetal dyad" concept in high-risk pregnancy;

3. Defining attributes: this step was carried out through integrative review. From it, the attributes associated with the NANDA-I taxonomy "disturbed maternal-fetal dyad" concept were identified, especially the recurring characteristics ${ }^{(4-5)}$. It is noteworthy that these attributes will be used to revise the "risk for disturbed maternal-fetal dyad" ND definition;

4. Identifying antecedents and consequences: it was also carried out through the integrative review. The antecedents and consequences that negatively affect the maternal-fetal dyad have been identified, which are risk factors, population at risk and related conditions ${ }^{(4-5)}$. They will be used to review the elements of the "risk for disturbed maternal-fetal dyad"ND;

5. Identifying a model case: a model case was identified to exemplify what the concept is. According to Walker and Avant $^{(5)}$, the case can be sought in literature, be real or constructed by the analyst;

6. Identifying borderline, related, and contrary cases: borderline, related, and contrary cases have been identified to exemplify what the concept is not. These cases can also be sought in literature, be real or constructed by the analyst ${ }^{(5)}$.

The integrative review was carried out with the purpose of operationalizing steps 3 and 4, as mentioned. This review went 
through six steps: 1 . Identifying the theme and the research question; 2. Sampling or literature search; 3. Defining the information to be extracted from the selected articles (categorization of studies); 4 . Assessing the studies included in the integrative review; 5 . Interpreting the results; 6 . Presenting the knowledge review or synthesis ${ }^{(6)}$.

Thus, the question that guided the integrative literature review was: what are the critical attributes and antecedents of the NANDA-I taxonomy "maternal-fetal dyad" concept in high-risk pregnancy?

\section{Search strategy}

To select the studies, a search was performed through access to PubMed, CINAHL, Scopus, LILACS and SciELO. The descriptors used are indexed in the Health Sciences Descriptors (DeCS) and in the Medical Subject Heading (MeSH) were "Pregnancy, High-Risk"; "Complications of pregnancy" and "Risk Factors", with the Boolean operator AND. Selected articles were published between 2008 and 2020.

\section{Inclusion and exclusion criteria}

Original and review articles were available, available electronically and in full, published in Portuguese, English or Spanish and which addressed the subject under study. Theses, dissertations and letters to the editor were excluded.

\section{Study selection process}

One thousand three hundred and forty-six studies were identified in databases and virtual library. Titles and abstracts were read. Of this total, five articles were excluded, as they were repeated between databases. Subsequently, 1,289 articles were excluded, as they did not meet the proposed criteria, totaling 52 articles to be read in full. Of the 52 articles, eight publications that did not address the attributes and background of the concept were excluded. At the end, 44 articles were selected to support the integrative review (Figure 1).

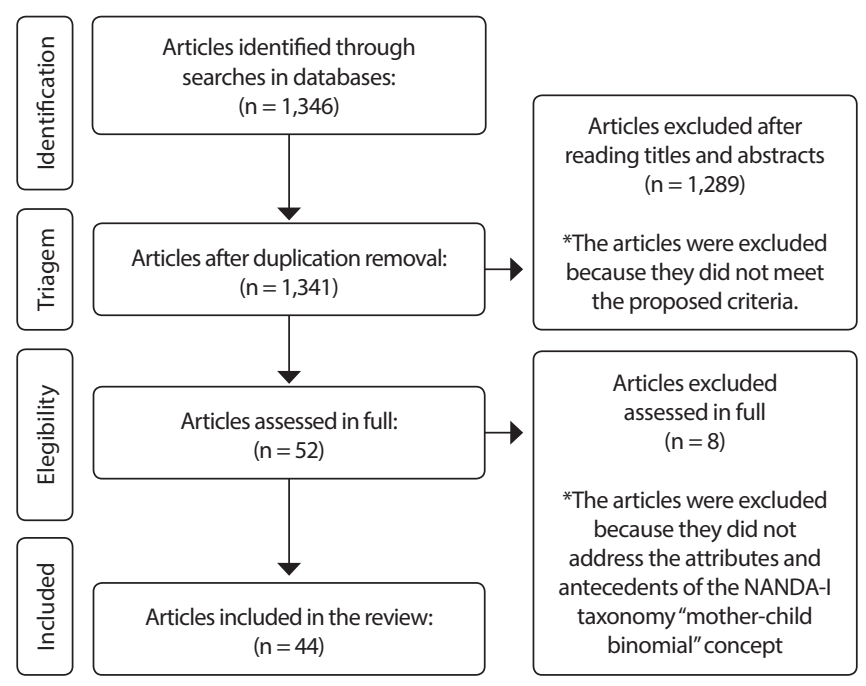

Figure 1 - Flowchart of study selection according to Preferred Reporting Items for Systematic Reviews and Meta-Analyzes (PRISMA), 2020

\section{Data extraction and assessment of the methodological quality of eligible studies}

Defining the information to be extracted from the selected articles was carried out using the data collection instrument developed for this research. The instrument contained the characterization of the studies, namely: title, authors, database, language, country, year and field of publication, objective, method, level of evidence (the evaluation was carried out using the evidence-based practices guide in nursing) ${ }^{(7)}$ and methodological rigor (the evaluation was carried out through the Critical Appraisal Skills Program (CASP) ${ }^{(8)}$ specific to each type of study). Moreover, the instrument made it possible to obtain the attributes and antecedents of the "maternal-fetal dyad" concept that were found.

\section{Analysis of data and interpretation of results}

The fourth step, assessing the studies included in the integrative review, aimed at analyzing the collected data. The fifth stage, interpreting the results, corresponded to the discussion of the main results, comparing them with the elements of the "risk for disturbed maternal-fetal dyad"ND. The review was presented in the last step.

\section{RESULTS}

The elements that characterize the concept (attributes) and antecedents (risk factors, population at risk and related conditions) were identified. To identify the antecedents, events that negatively affect the maternal-fetal dyad were analyzed (Chart 1).

Furthermore, it should be noted that all articles in the review were classified in category $A$ ( 6 to 10 points) for methodological rigor, which are studies with good methodological quality and minimized bias $(n=44)^{(9-52)}$. Most articles were published between 2013 and $2015(n=22)^{(9-11,15-17,19-21,23-32,40-42)}$ and in non-nursing journals $(n=41)^{(12-18,20-53)}$. The articles in English $(n=30)^{(9-16,18-31,41,46-52)}$, published in the United States of America $(n=12)^{(10-16,18-19,22,24,26)}$ and with evidence level IV - well-outlined cohort and case-control studies stood out $(n=17)^{(14,16-18,21-25,31-32,34,36-37,40-41,43,46-48,50)}$. Most were selected mainly from the Scopus database $(n=16)^{(17-32)}$.

Of the two attributes found, only "symbiotic maternal-fetal relationship" is in NAND-I's definition of the diagnosis under study.

Of the ten identified risk factors, five are included in the NANDA-I taxonomy ${ }^{(4)}$, despite using different nomenclature such as tobacco, alcohol and drugs; violence and absent/inadequate prenatal care. Analysis allowed identifying other risk factors of the diagnosis, which are not included in NANDA-I such as overweight, obesity, low gestational weight gain, absent/inadequate partner support, and absent/inadequate social support.

Four related conditions (complications of pregnancy, impaired glucose metabolism, medication regimen and compromised oxygen transfer) are included in NANDA-I ${ }^{(4)}$ and two are not (diseases and maternal conditions). None of the population at risk found in this study is in that taxonomy.

Furthermore, the model case and the opposite case were identified, which were adapted from the literature ${ }^{(16)}$ (Charts 2 and 3). It is noteworthy that these cases will show the representation of the concept and what the concept is not, respectively. 
Chart 1 - Distribution of studies included in the integrative review according to title, year, atributes, and antecedents, 2020

\begin{tabular}{|c|c|c|c|}
\hline Title & Year & Attributes & Antecedents \\
\hline $\begin{array}{l}\text { Pre-pregnancy } \\
\text { counselling for } \\
\text { women with chronic } \\
\text { kidney disease: } \\
\text { a retrospective } \\
\text { analysis of nine years' } \\
\text { experience } e^{(9)}\end{array}$ & 2015 & - & $\begin{array}{l}\text { Related conditions: } \\
\text { complications } \\
\text { of pregnancy; } \\
\text { compromised oxygen } \\
\text { transfer; medication } \\
\text { regimen. }\end{array}$ \\
\hline $\begin{array}{l}\text { Preeclampsia in } \\
\text { high risk women } \\
\text { is characterized by } \\
\text { risk group-specific } \\
\text { abnormalities in } \\
\text { serum biomarkers }{ }^{(10)}\end{array}$ & 2014 & - & $\begin{array}{l}\text { Related conditions: } \\
\text { complications } \\
\text { of pregnancy; } \\
\text { compromised oxygen } \\
\text { transfer; maternal } \\
\text { illnesses. }\end{array}$ \\
\hline $\begin{array}{l}\text { Complications of } \\
\text { pregnancy and } \\
\text { Cardiovascular } \\
\text { Disease Death: Fifty- } \\
\text { Year Follow-Up of } \\
\text { the Child Health and } \\
\text { Development Studies } \\
\text { Pregnancy Cohort }{ }^{(11)}\end{array}$ & 2015 & $\begin{array}{lll}- & - \\
-1 & -1\end{array}$ & $\begin{array}{l}\text { Risk factors: overweight } \\
\text { and tobacco. } \\
\\
\text { Population at risk: } \\
\text { preeclampsia in previous } \\
\text { pregnancy. } \\
\\
\text { Related conditions: } \\
\text { complications } \\
\text { of pregnancy; } \\
\text { compromised oxygen } \\
\text { transfer; maternal } \\
\text { illnesses. }\end{array}$ \\
\hline $\begin{array}{l}\text { Understanding } \\
\text { the association } \\
\text { of biomedical, } \\
\text { psychosocial and } \\
\text { behavioral risks with } \\
\text { adverse pregnancy }\end{array}$ & 2011 & 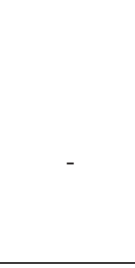 & $\begin{array}{l}\text { Risk factors: overweight; } \\
\text { tobacco; violence; drugs; } \\
\text { alcohol. } \\
\text { Related conditions: } \\
\text { compromised oxygen } \\
\text { transfer and maternal } \\
\text { illnesses. } \\
\end{array}$ \\
\hline $\begin{array}{l}\text { Effect of older } \\
\text { maternal age on the } \\
\text { risk of spontaneous } \\
\text { preterm labor: a } \\
\text { population-based } \\
\text { study }^{(13)}\end{array}$ & 2009 & - & $\begin{array}{l}\text { Risk factors: tobacco; } \\
\text { drugs; alcohol; low } \\
\text { gestational weight gain. } \\
\text { Population at risk: } \\
\text { increased maternal age. } \\
\\
\text { Related conditions: } \\
\text { complications } \\
\text { of pregnancy; } \\
\text { compromised oxygen } \\
\text { transfer; maternal } \\
\text { illnesses. }\end{array}$ \\
\hline $\begin{array}{l}\text { Maternal obesity } \\
\text { in early pregnancy } \\
\text { and risk of adverse } \\
\text { outcomes }^{(14)}\end{array}$ & 2013 & - & Risk factors: obesity. \\
\hline $\begin{array}{l}\text { Pregnancy } \\
\text { characteristics and } \\
\text { women's future } \\
\text { cardiovascular } \\
\text { health: an underused } \\
\text { opportunity to } \\
\text { improve women's } \\
\text { health? }\end{array}$ & 2013 & $\begin{array}{lll}- & -1 \\
\end{array}$ & $\begin{array}{l}\text { Risk factors: tobacco and } \\
\text { obesity. } \\
\text { Population at risk: } \\
\text { increased maternal age. } \\
\text { Related conditions: } \\
\text { complications } \\
\text { of pregnancy; } \\
\text { compromised oxygen } \\
\text { transfer; maternal } \\
\text { illnesses; maternal } \\
\text { conditions. }\end{array}$ \\
\hline
\end{tabular}

Chart

\begin{tabular}{|c|c|c|c|}
\hline Title & Year & Attributes & Antecedents \\
\hline $\begin{array}{l}\text { Maternal risk during } \\
\text { pregnancy: a concept } \\
\text { analysis }^{(16)}\end{array}$ & 2016 & - & Risk factors: drugs. \\
\hline $\begin{array}{l}\text { Deficiência de } \\
\text { vitamina D na } \\
\text { gravidez e o seu } \\
\text { impacto sobre o feto, } \\
\text { o recém-nascido e na } \\
\text { infância }^{(17)}\end{array}$ & 2015 & $\begin{array}{l}\text { Maternal- } \\
\text { fetal bond; } \\
\text { symbiotic } \\
\text { maternal- } \\
\text { fetal } \\
\text { relationship. }\end{array}$ & $\begin{array}{l}\text { Related conditions: } \\
\text { complications } \\
\text { of pregnancy; } \\
\text { compromised oxygen } \\
\text { transfer; maternal } \\
\text { illnesses; maternal } \\
\text { conditions. }\end{array}$ \\
\hline $\begin{array}{l}\text { Effect of maternal } \\
\text { moderate to severe } \\
\text { asthma on perinatal } \\
\text { outcomes }^{(18)}\end{array}$ & 2010 & - & $\begin{array}{l}\text { Related conditions: } \\
\text { compromised oxygen } \\
\text { transfer. }\end{array}$ \\
\hline $\begin{array}{l}\text { Gestational weight } \\
\text { gain and risks for } \\
\text { adverse perinatal } \\
\text { outcomes: A } \\
\text { retrospective cohort } \\
\text { study based on } \\
\text { the } 2009 \text { Institute } \\
\text { of Medicine } \\
\text { guidelines }^{(19)}\end{array}$ & 2015 & - & $\begin{array}{l}\text { Risk factors: overweight } \\
\text { and obesity. } \\
\text { Related conditions: } \\
\text { complications of } \\
\text { pregnancy; maternal } \\
\text { illnesses; maternal } \\
\text { conditions. }\end{array}$ \\
\hline $\begin{array}{l}\text { Obstetrical } \\
\text { complications } \\
\text { in women with } \\
\text { epilepsy }\end{array}$ & 2015 & - & $\begin{array}{l}\text { Related conditions: } \\
\text { compromised oxygen } \\
\text { transfer and medication } \\
\text { regimen. }\end{array}$ \\
\hline $\begin{array}{l}\text { Intrauterine growth } \\
\text { retardation in } \\
\text { foetuses of women } \\
\text { with epilepsy }\end{array}$ & 2015 & - & $\begin{array}{l}\text { Risk factors: tobacco. } \\
\text { Related conditions: } \\
\text { complications } \\
\text { of pregnancy; } \\
\text { compromised oxygen } \\
\text { transfer; medication } \\
\text { regimen. }\end{array}$ \\
\hline $\begin{array}{l}\text { Risk factors for } \\
\text { congenital anomalies } \\
\text { in high risk pregnant } \\
\text { women: A large study } \\
\text { from South India } \\
\text { (22) }\end{array}$ & 2017 & - & $\begin{array}{l}\text { Risk factors: absent/ } \\
\text { inadequate prenatal } \\
\text { care. } \\
\text { Related conditions: } \\
\text { complications of } \\
\text { pregnancy. }\end{array}$ \\
\hline $\begin{array}{l}\text { Maternal overweight } \\
\text { and obesity and risk } \\
\text { of pre-eclampsia in } \\
\text { women with type } 1 \\
\text { diabetes or type } 2 \\
\text { diabetes }^{(23)}\end{array}$ & 2016 & - & $\begin{array}{l}\text { Population at risk: } \\
\text { preeclampsia in previous } \\
\text { pregnancy. } \\
\text { Related conditions: } \\
\text { maternal illnesses. }\end{array}$ \\
\hline $\begin{array}{l}\text { Body mass index, } \\
\text { gestational weight } \\
\text { gain, and obstetric } \\
\text { complications } \\
\text { in Moroccan } \\
\text { population }^{(24)}\end{array}$ & 2013 & - & $\begin{array}{l}\text { Risk factors: overweight } \\
\text { and obesity. } \\
\text { Related conditions: } \\
\text { compromised oxygen } \\
\text { transfer and maternal } \\
\text { illnesses. }\end{array}$ \\
\hline $\begin{array}{l}\text { Early gestational } \\
\text { weight gain rate and } \\
\text { adverse pregnancy } \\
\text { outcomes in Korean } \\
\text { women }^{(25)}\end{array}$ & 2015 & $\begin{array}{l}\text { Symbiotic } \\
\text { maternal- } \\
\text { fetal } \\
\text { relationship. }\end{array}$ & $\begin{array}{l}\text { Risk factors: obesity. } \\
\text { Population at risk: } \\
\text { increased maternal age. }\end{array}$ \\
\hline $\begin{array}{l}\text { Pregnancy at very } \\
\text { increased maternal } \\
\text { age: a UK population } \\
\text { based cohort study }{ }^{(26)}\end{array}$ & 2017 & - & $\begin{array}{l}\text { Related conditions: } \\
\text { maternal illnesses. }\end{array}$ \\
\hline
\end{tabular}


Chart 1

\begin{tabular}{|c|c|c|c|}
\hline Title & Year & Attributes & Antecedents \\
\hline $\begin{array}{l}\text { Pre-pregnancy } \\
\text { high-risk factors at } \\
\text { first antenatal visit: } \\
\text { how predictive are } \\
\text { these of pregnancy } \\
\text { outcomes?(27) }\end{array}$ & 2014 & - & $\begin{array}{l}\text { Risk factors: overweight; } \\
\text { tobacco; obesity; absent/ } \\
\text { inadequate prenatal care. } \\
\text { Population at risk: } \\
\text { increased maternal age. } \\
\text { Related conditions: } \\
\text { complications of } \\
\text { pregnancy; compromised } \\
\text { oxygen transfer; maternal } \\
\text { illnesses. }\end{array}$ \\
\hline $\begin{array}{l}\text { Maternal obesity } \\
\text { in early pregnancy } \\
\text { and risk of adverse } \\
\text { outcome }^{(28)}\end{array}$ & 2013 & - & $\begin{array}{l}\text { Risk factors: overweight; } \\
\text { drugs; alcohol; obesity. } \\
\text { Related conditions: } \\
\text { complications of } \\
\text { pregnancy and maternal } \\
\text { illnesses. }\end{array}$ \\
\hline $\begin{array}{l}\text { Obstetrical } \\
\text { complications } \\
\text { in women with } \\
\text { endometriosis: a } \\
\text { cohort study in } \\
\text { Japan }^{(29)}\end{array}$ & 2016 & - & $\begin{array}{l}\text { Related conditions: } \\
\text { complications of } \\
\text { pregnancy; compromised } \\
\text { oxygen transfer; maternal } \\
\text { illnesses; maternal } \\
\text { conditions. }\end{array}$ \\
\hline $\begin{array}{l}\text { Patients with } \\
\text { subclinical } \\
\text { hypothyroidism } \\
\text { before } 20 \text { weeks } \\
\text { of pregnancy } \\
\text { have a higher risk } \\
\text { of miscarriage: a } \\
\text { systematic review and } \\
\text { meta-analysis }^{(30)}\end{array}$ & 2017 & - & $\begin{array}{l}\text { Related conditions: } \\
\text { maternal illnesses. }\end{array}$ \\
\hline $\begin{array}{l}\text { Risk factors and birth } \\
\text { outcomes of anaemia } \\
\text { in early pregnancy in } \\
\text { a nulliparous cohort }{ }^{(31)}\end{array}$ & 2015 & - & $\begin{array}{l}\text { Related conditions: } \\
\text { complications of } \\
\text { pregnancy and } \\
\text { compromised oxygen } \\
\text { transfer. }\end{array}$ \\
\hline $\begin{array}{l}\text { Weight change } \\
\text { across the start of } \\
\text { three consecutive } \\
\text { pregnancies and } \\
\text { the risk of maternal } \\
\text { morbidity and SGA } \\
\text { birth at the second } \\
\text { and third pregnancy }\end{array}$ & 2017 & - & $\begin{array}{l}\text { Risk factors: overweight } \\
\text { and obesity. } \\
\text { Related conditions: } \\
\text { maternal illnesses. }\end{array}$ \\
\hline $\begin{array}{l}\text { Morbidade materna } \\
\text { grave em um hospital } \\
\text { universitário de } \\
\text { referência municipal } \\
\text { em Campinas, Estado } \\
\text { de São Paulo }\end{array}$ & 2008 & - & $\begin{array}{l}\text { Related conditions: } \\
\text { complications of } \\
\text { pregnancy. }\end{array}$ \\
\hline $\begin{array}{l}\text { Estudo retrospectivo } \\
\text { das implicações } \\
\text { maternas, fetais e } \\
\text { perinatais em mulheres } \\
\text { portadoras de } \\
\text { diabetes, em } 20 \text { anos } \\
\text { de acompanhamento } \\
\text { no Hospital Escola da } \\
\text { Universidade Federal } \\
\text { do Triângulo Mineiro }{ }^{(34)}\end{array}$ & 2010 & $\begin{array}{l}\text { Maternal- } \\
\text { fetal bond. }\end{array}$ & $\begin{array}{l}\text { Risk factors: tobacco. } \\
\text { Related conditions: } \\
\text { maternal illnesses. }\end{array}$ \\
\hline $\begin{array}{l}\text { Aspectos } \\
\text { sociodemográficos } \\
\text { y obstétricos de la } \\
\text { morbilidad materna } \\
\text { grave }^{(35)}\end{array}$ & 2017 & - & $\begin{array}{l}\text { Related conditions: } \\
\text { complications of } \\
\text { pregnancy and } \\
\text { compromised oxygen } \\
\text { transfer. }\end{array}$ \\
\hline
\end{tabular}

Chart

\begin{tabular}{|c|c|c|c|}
\hline Title & Year & Attributes & Antecedents \\
\hline $\begin{array}{l}\text { Morbidade materna } \\
\text { grave na microrregião } \\
\text { de Barbacena/MG }{ }^{(36)}\end{array}$ & 2016 & - & $\begin{array}{l}\text { Risk factors: absent/ } \\
\text { inadequate prenatal care. } \\
\text { Population at risk: } \\
\text { increased maternal age. } \\
\text { Related conditions: } \\
\text { complications } \\
\text { of pregnancy; } \\
\text { compromised oxygen } \\
\text { transfer; maternal } \\
\text { illnesses. }\end{array}$ \\
\hline $\begin{array}{l}\text { Gestações } \\
\text { complicadas por } \\
\text { sífilis materna e óbito } \\
\text { fetall }^{(37)}\end{array}$ & 2011 & - & $\begin{array}{l}\text { Related conditions: } \\
\text { complications of } \\
\text { pregnancy; maternal } \\
\text { illnesses; maternal } \\
\text { conditions. }\end{array}$ \\
\hline $\begin{array}{l}\text { Influência do estado } \\
\text { nutricional materno, } \\
\text { ganho de peso e } \\
\text { consumo energético } \\
\text { sobre o crescimento } \\
\text { fetal, em gestações de } \\
\text { alto risco }{ }^{(38)}\end{array}$ & 2017 & $\begin{array}{l}\text { Maternal- } \\
\text { fetal bond. }\end{array}$ & $\begin{array}{l}\text { Risk factors: obesity. } \\
\text { Related conditions: } \\
\text { compromised oxygen } \\
\text { transfer and maternal } \\
\text { illnesses. }\end{array}$ \\
\hline $\begin{array}{l}\text { Muerte fetal: } \\
\text { caracterización } \\
\text { epidemiológica(39) }^{(39}\end{array}$ & 2016 & $\begin{array}{l}\text { Maternal- } \\
\text { fetal bond. }\end{array}$ & $\begin{array}{l}\text { Risk factors: overweight; } \\
\text { tobacco; obesity. } \\
\text { Related conditions: } \\
\text { complications of } \\
\text { pregnancy; compromised } \\
\text { oxygen transfer; maternal } \\
\text { illnesses. }\end{array}$ \\
\hline $\begin{array}{l}\text { Estrés psicosocial } \\
\text { laboral como factor } \\
\text { de riesgo para las } \\
\text { complicaciones de la } \\
\text { gestación y el bajo peso }^{\text {al nacer }^{(40)}}\end{array}$ & 2013 & $\begin{array}{l}\text { Maternal- } \\
\text { fetal bond. }\end{array}$ & $\begin{array}{l}\text { Related conditions: } \\
\text { complications of } \\
\text { pregnancy and } \\
\text { compromised oxygen } \\
\text { transfer. }\end{array}$ \\
\hline $\begin{array}{l}\text { Depressive } \\
\text { symptoms in } \\
\text { pregnancy and } \\
\text { associated factors: } \\
\text { longitudinal study }{ }^{(41)}\end{array}$ & 2017 & $\begin{array}{l}\text { Maternal- } \\
\text { fetal bond. }\end{array}$ & $\begin{array}{l}\text { Risk factors: absent/ } \\
\text { inadequate partner } \\
\text { support and absent/ } \\
\text { inadequate social } \\
\text { support. } \\
\text { Population at risk: } \\
\text { unintended pregnancy; } \\
\text { low education } \\
\text { level; economically } \\
\text { disadvantaged. } \\
\text { Related conditions: } \\
\text { compromised oxygen } \\
\text { transfer. }\end{array}$ \\
\hline $\begin{array}{l}\text { Influencia de } \\
\text { la obesidad } \\
\text { pregestacional } \\
\text { en el riesgo de } \\
\text { preeclampsial } \\
\text { eclampsia }^{(42)}\end{array}$ & 2013 & - & $\begin{array}{l}\text { Risk factors: obesity. } \\
\text { Related conditions: } \\
\text { compromised oxygen } \\
\text { transfer. }\end{array}$ \\
\hline $\begin{array}{l}\text { Mortalidad Perinatal } \\
\text { de la Población } \\
\text { afiliada a una EPS de } \\
\text { Pasto. Departamento } \\
\text { de Nariño }{ }^{(43)}\end{array}$ & 2015 & - & $\begin{array}{l}\text { Risk factors: tobacco; } \\
\text { alcohol; absent/ } \\
\text { inadequate prenatal care. } \\
\text { Related conditions: } \\
\text { complications of } \\
\text { pregnancy; compromised } \\
\text { oxygen transfer; maternal } \\
\text { illnesses. }\end{array}$ \\
\hline
\end{tabular}


Chart 1

\begin{tabular}{|c|c|c|c|}
\hline Title & Year & Attributes & Antecedents \\
\hline $\begin{array}{l}\text { Complicações } \\
\text { maternas em } \\
\text { gestantes com idade } \\
\text { avançada }{ }^{(44)}\end{array}$ & 2012 & - & $\begin{array}{l}\text { Risk factors: tobacco } \\
\text { and absent/inadequate } \\
\text { prenatal care. } \\
\text { Related conditions: } \\
\text { complications of } \\
\text { pregnancy; impaired } \\
\text { glucose metabolism; } \\
\text { compromised oxygen } \\
\text { transfer; maternal illnesses. }\end{array}$ \\
\hline $\begin{array}{l}\text { Embarazo de alto } \\
\text { riesgo }^{(45)}\end{array}$ & 2012 & - & $\begin{array}{l}\text { Risk factors: drugs; alcohol; } \\
\text { obesity. } \\
\text { Population at risk: } \\
\text { low education level } \\
\text { and economically } \\
\text { disadvantaged. } \\
\text { Related conditions: } \\
\text { compromised oxygen } \\
\text { transfer and maternal } \\
\text { illnesses. }\end{array}$ \\
\hline $\begin{array}{l}\text { Prevalence and } \\
\text { profile of adverse } \\
\text { drug reactions in } \\
\text { high-risk pregnancy: } \\
\text { a cohort study }\end{array}$ & 2019 & - & $\begin{array}{l}\text { Related conditions: } \\
\text { impaired glucose } \\
\text { metabolism; maternal } \\
\text { illnesses; medication } \\
\text { regimen. }\end{array}$ \\
\hline $\begin{array}{l}\text { Maternal near miss } \\
\text { determinants at a } \\
\text { maternity hospital for } \\
\text { high-risk pregnancy } \\
\text { in northeastern } \\
\text { Brazil: a prospective } \\
\text { study }(47)\end{array}$ & 2018 & - & $\begin{array}{l}\text { Risk factors: absent/ } \\
\text { inadequate prenatal care. } \\
\text { Population at risk: low } \\
\text { education level and } \\
\text { increased maternal age. } \\
\text { Related conditions: } \\
\text { compromised oxygen } \\
\text { transfer; maternal } \\
\text { illnesses; complications of } \\
\text { pregnancy. }\end{array}$ \\
\hline $\begin{array}{l}\text { High risk pregnancy } \\
\text { associated perinatal } \\
\text { morbidity and } \\
\text { mortality: a second } \\
\text { birth } \\
\text { population-based } \\
\text { survey in Huai'an in } \\
2015^{(48)}\end{array}$ & 2019 & $\begin{array}{c}\text { Symbiotic } \\
\text { maternal- } \\
\text { fetal } \\
\text { relationship. }\end{array}$ & $\begin{array}{l}\text { Population at risk: low } \\
\text { education level and } \\
\text { increased maternal age. } \\
\text { Related conditions: } \\
\text { maternal illnesses } \\
\text { and complications of } \\
\text { pregnancy. }\end{array}$ \\
\hline $\begin{array}{l}\text { Complications and } \\
\text { pre-existing diseases } \\
\text { in pregnant women } \\
\text { with Diabetes } \\
\text { mellitus }^{(49)}\end{array}$ & 2019 & - & $\begin{array}{l}\text { Risk factors: obesity and } \\
\text { tobacco. } \\
\text { Population at risk: } \\
\text { increased maternal age } \\
\text { and preeclampsia in } \\
\text { previous pregnancy. } \\
\text { Related conditions: } \\
\text { impaired glucose } \\
\text { metabolism and maternal } \\
\text { illnesses. }\end{array}$ \\
\hline $\begin{array}{l}\text { Gestational diabetes } \\
\text { and pre-natal } \\
\text { assistance at high } \\
\text { risk }^{(50)}\end{array}$ & 2019 & - & $\begin{array}{l}\text { Risk factors: overweight; } \\
\text { obesity; absent/ } \\
\text { inadequate prenatal care. } \\
\text { Population at risk: } \\
\text { increased maternal age. } \\
\text { Related conditions: } \\
\text { impaired glucose } \\
\text { metabolism and } \\
\text { maternal illnesses. }\end{array}$ \\
\hline
\end{tabular}

To be continued

\begin{tabular}{|c|c|c|c|}
\hline Title & Year & Attributes & Antecedents \\
\hline $\begin{array}{l}\text { Analysis of neonatal } \\
\text { mortality risk } \\
\text { factors in Brazil: a } \\
\text { systematic review } \\
\text { and meta-analysis } \\
\text { of observational } \\
\text { studies }^{(51)}\end{array}$ & 2019 & $\begin{array}{l}\text { Maternal- } \\
\text { fetal bond. }\end{array}$ & $\begin{array}{l}\text { Risk factors: absent/ } \\
\text { inadequate prenatal } \\
\text { care. } \\
\text { Population at risk: low } \\
\text { education level and } \\
\text { increased maternal age. } \\
\text { Related conditions: } \\
\text { complications of } \\
\text { pregnancy. }\end{array}$ \\
\hline $\begin{array}{l}\text { Influence of maternal } \\
\text { age in perinatal } \\
\text { conditions } \\
\text { In live births of São } \\
\text { Luís, Maranhão(52) }\end{array}$ & 2020 & - & $\begin{array}{l}\text { Risk factors: absent/ } \\
\text { inadequate prenatal care. } \\
\text { Population at risk: low } \\
\text { education level and } \\
\text { increased maternal age. }\end{array}$ \\
\hline
\end{tabular}

Chart 2 - Exemplification of the model case of the "maternal-fetal dyad" concept, 2020

\section{Model case}

R.D., 31 years old, primigravida, starts prenatal consultations at eight weeks of gestation with the purpose of maintaining an efficient monitoring and quality assistance for the maternal-fetal dyad. Although she perceives herself to be healthy, she worries about pregnancy risk factors, as she is aware of the symbiotic maternal-fetal relationship. In prenatal consultations, R.D. increases the knowledge about the well-being of the dyad, as she starts to realize how essential the bond between her and her child is to maintain the physiological exchange and the good quality of affective-emotional interactions during the gestational period. ${ }^{(16)}$.

Chart 3 - Exemplification of the contrary case with antecedents that affect the maternal-fetal dyad, 2020

\section{Contrary case}

M.V. is a 36-year-old primigravida and starts labor at 28 weeks of gestation due to complications of pregnancy. She reports not having had prenatal care, complains of headache, epigastric pain and is concerned about the well-being of the child. The nurse performs fetal monitoring, obtains the vital signs of M.V and checks the presence of protein in the urine, to observe the presence or absence of maternal and fetal complications. Blood pressure is $170 / 98 \mathrm{mmHg}$ and proteinuria is observed in the Labstix test $(2+)$. The nurse identifies the potential for pre-eclampsia and subsequent fetal hypoxia, which can lead to compromised maternal and fetal health. M.V. claims that she had headaches throughout her pregnancy. The nurse, as well as the doctor, discusses this situation with M.V., providing information about her condition that contributes to the risk for disturbed maternal-fetal dyad and the potential adverse effects on her health and that of her child ${ }^{(16)}$.

\section{DISCUSSION}

It is observed in the model case how the concept "maternal-fetal dyad" is defined through the attributes found in the literature. Meanwhile, it is observed how the concept cannot be defined in the contrary case. There are antecedents that affect the maternal-fetal dyad, among them: increased maternal age, absent prenatal care, premature labor, preeclampsia, proteinuria and compromised oxygen transport to the child. These complications of pregnancy caused maternal and fetal losses, resulting in the risk for disturbed maternal-fetal dyad ${ }^{(44-45)}$.

The attributes of the "maternal-fetal dyad" concept that stood out in literature were: maternal-fetal bond and symbiotic 
maternal-fetal relationship. The NANDA-I's definition for the diagnosis under study is "at risk for disruption of the symbiotic maternal-fetal dyad as a result of comorbidity or pregnancy-related conditions"(4). Therefore, it is suggested that the definition needs to be more consistent considering the impairment of physiological exchange and affective-emotional interactions: "at risk for disruption of the symbiotic maternal-fetal dyad as a result of comorbidity or pregnancy-related conditions".

Negative pregnancy outcomes are strongly influenced by risk factors. Such risk factors trigger problems during pregnancy, making the mother and fetus vulnerable to complications and morbidities $^{(44-52)}$.

The NANDA-I "substance abuse" risk factor was identified as tobacco, drugs and alcohol. These factors during pregnancy generate compromised health for the woman and the fetus and are related to the etiology of premature birth, spontaneous abortions and fetal malformations ${ }^{(45)}$. It is noteworthy that in Brazil, in 2016, the estimated prevalence of the use of these substances during pregnancy was $1.45 \%$ for drugs, $22.32 \%$ for alcohol and $4.22 \%$ for tobacco ${ }^{(53)}$. It is observed that alcohol is the most prevalent in pregnancy.

"Absent/inadequate partner support" and "absent/inadequate social support" risk factors were also found in nursing literature, but they are not included in the NANDA-I taxonomy. According to nursing literature, lack of support and non-acceptance of the partner during pregnancy are the leading causes of symptoms of depression. Approximately $10 \%$ of pregnant women have symptoms of depression, mild or moderate, which can interfere with fetal development and increase risks to both the health of the mother and the fetus ${ }^{(41)}$. In Brazil, there were $25 \%$ of pregnant women with depression in $2017 \%{ }^{(53)}$.

Analysis of the elements of the ND allowed identification of other risk factors that are not included in NANDA-l: overweight, obesity and low gestational weight gain. It is known that the altered maternal weight is one of the contributors for the development of complications of pregnancy, since the maternal nutritional status before and during pregnancy has a significant influence on maternal health and fetal development ${ }^{(42)}$. In Brazil, in 2017, $26.55 \%$ of pregnant women were overweight, $15 \%$ were obese and $17.34 \%$ were underweight ${ }^{(53)}$.

Overweight and obesity are increasingly present in pregnancy and can lead to gestational diabetes, pre-eclampsia, fetal mortality, congenital malformations, premature birth, macrosomia and polyhydramnios. Low maternal weight can restrict fetal growth ${ }^{(42)}$.

As for the risk factor "absent/inadequate prenatal care", NANDA-I only mentions the term "inappropriate", when it should also include the term "absent", as mentioned in nursing literature, since in 2016, in Brazil, 551,369 pregnant women had an inadequate number of prenatal consultations and 15,636 pregnant women did not attend them ${ }^{(53)}$. Absence or difficulty in accessing prenatal care may be related to socioeconomic, accessibility, quality of care and social support, maternal age, alcohol and other drugs, multiparity, non-acceptance of pregnancy, lack of family support and lack of connection with health services factors. It should be noted that failure to perform prenatal care can directly affect maternal and/or fetal health ${ }^{(53)}$.

It should be noted that NANDA-I does not present, for the diagnosis under study, the clinical indicator "population at risk".
However, in this conceptual analysis, five population at risk were identified: increased maternal age; preeclampsia in previous pregnancy; unintended pregnancy; low education level and economically disadvantaged. These populations are more likely to develop the diagnosis of risk for disturbed maternal-fetal dyad ${ }^{(44,53)}$.

Women who do not plan pregnancy, as well as pregnant women who have low education level and/or low socioeconomic status, are also susceptible to unfavorable pregnancy outcomes, such as pre-eclampsia, premature labor and low birth weight, a once compromise maternal-fetal bond ${ }^{(41,53)}$.

Pregnant women with increased maternal age ( $\geq 35$ years old) are more predisposed to maternal and fetal morbidity and the risk of maternal mortality ${ }^{(44)}$. Furthermore, they have greater complications of pregnancy such as gestational hypertension, gestational diabetes mellitus, premature labor, placenta previa or abruption, premature rupture of membranes, and multiple gestations. In Brazil, in 2010, 299,372 women aged 35 and over got pregnant. In 2016, this value increased by $22.14 \%$, which is equivalent to 384,496 pregnant women with increased maternal age ${ }^{(53)}$.

The results of this review mentioned that gestational risk is observed in women with increased maternal age, however early maternal age can be a determining factor for gestational risk ${ }^{(44-45)}$.

Concerning related conditions, which are not changeable by nurses, six were found in nursing literature. Among them, diseases and maternal conditions are not included in the taxonomy, which threatens $20 \%$ of pregnancies, making them at high risk. Such diseases and maternal conditions are pre-existing hypertension, depression, dyslipidemia, systemic inflammation, vitamin D deficiency, maternal infections, autoimmune diseases, thyroid dysfunction and endometriosis. They cause complications of pregnancy, namely: hemorrhages, pre-eclampsia, unfavorable obstetric results, restriction of fetal development, premature birth, macrosomia, placenta previa, spontaneous abortion, fetal death, among others ${ }^{(52)}$.

The elements evidenced in the literature will assist nurses in the correct identification of the ND and in advising pregnant women about the potential problems that may arise during the gestational period. Complications of pregnancy have important long-term implications for both the health of the mother and the health of the child ${ }^{(52)}$. Nurses must also appropriate this knowledge in the decision-making process and in carrying out specific care for high-risk pregnant women. The correct identification of the factors responsible for the risk for disturbed maternal-fetal dyad favors the subsequent implementation of preventive and health promotion actions capable of intervening in complications of pregnancy ${ }^{(51-52)}$.

In the time frame used for this research, no studies were found in the literature that addressed the analysis of the risk concept of disturbed maternal-fetal dyad. There are only studies that cite the diagnosis in question in high-risk pregnant women.

\section{Study limitations}

As limitations of the research, it has been that the concept may be implicit in the literature, as well as unnecessary defining attributes and antecedents may have been included, establishing barriers for an effective analysis. Furthermore, conceptual analysis requires interpretation, which may reflect the researcher's subjectivity. 


\section{Contributions to nursing}

This study contributes to language development in the nursing field by identifying the risk factors of the laughing population and the related conditions of the ND risk for disturbed maternal-fetal dyad; this occurred in the context of high-risk pregnancy for the use of appropriate terminology; and this is indispensable for professional nursing practice.

\section{CONCLUSION}

The concept analysis allowed to identify the attributes and antecedents of the maternal-fetal dyad concept in high-risk pregnancy in the established time frame, raising and clarifying ideas. It was possible to identify 12 antecedents that are not included in NANDA-I. There was a need to review the definition of the diagnosis proposed by the taxonomy.

Further studies are recommended, especially in nursing, on understanding the concept, as most articles that met the inclusion criteria come from other field of health. It is necessary to review the risk factors; new components of the diagnosis proposed by the taxonomy (related conditions and populations at risk); conducting content analysis by experts so that experts can corroborate or not the results identified in this concept analysis. The diagnosis review will support a better direction in nursing care for pregnant women through actions to prevent diagnostic risk factors.

\section{REFERENCES}

1. Rodrigues ARM, Dantas SLC, Pereira AMM, Silveira MAMD, Rodrigues DP. Gravidez de alto risco: análise dos determinantes de saúde. Sanare[Internet]. 2017 [cited 2018 Jun 25];16(1):23-8. Available from: https://sanare.emnuvens.com.br/sanare/article/viewFile/1135/6202

2. Holness N. High Risk Pregnancy. Nur Clin North Am. 2018;53(2):241-51. doi: 10.1016/j.cnur.2018.01.0 10

3. Benedet SA, Gelbcke FL, Amante LN. Processo de enfermagem: instrumento da sistematização da assistência de enfermagem na percepção dos enfermeiros. Rev Pesqui: Cuid Fundam. 2016;8(3):4780-8. doi: 10.9789/2175-5361.2016.v8i3.4780-4788

4. Herdman H, Kamitsuru S. Diagnósticos de enfermagem da NANDA: definições e classificação 2018- 2020. Porto Alegre: Artmed; 2018.

5. Walker LO, Avant KC. Strategies for theory construction in nursing. Upper Saddle River: Pearson Prentice Hall; 2011.

6. Pompeo DA, Rossi LA, Galvão CM. Revisão Integrativa: etapa inicial do processo de validação de diagnóstico de enfermagem. Acta Paul Enferm. 2009;22(4):434-8. doi: 10.1590/S0103-21002009000400014

7. Melnyk BM, Fineout-Overholt E. Evidence-based practice in nursing \& healthcare: A guide to best practice. New York: Lippincott Williams \& Wilkins; 2011.

8. Critical Appraisal Skills Programm. Critical Appraisal Checklists [Internet]. Oxford: CASP; 2018 [cited 2018 Jun 27]. Available from: https:// casp-uk.net/casp-tools-checklists/

9. Wiles KS, Kate B, Alina V, Kate RH, Paramit C, Cath JT, Catherine N-P. Pre-pregnancy counselling for women with chronic kidney disease: a retrospective e analysis of nine years' experience. BMC Nephrol. 2015;16(1):16-28. doi: 10.1186/s12882-015-0024-6

10. Metz TD, Allshouse AA, Euser AG, Kent DH. Preeclampsia in high risk women is characterized by risk group specific abnormalities in serum biomarkers. Am J Obstet Gynecol. 2014;211(5):512-6. doi: 10.1016/j.ajog.2014.04.027

11. Cirillo PM, Cohn BA. Pregnancy complications and cardiovascular disease death: fifty year follow up of the child health and development studies pregnancy cohort. Circul AHA. 2015;1(1):1-24. doi: 10.1161/CIRCULATIONAHA.113.003901

12. Kiely M, Ayman A, Gantz MG, Chowdhury D, Thornberry JS, El-Khorazaty MN. Understanding the association of biomedical, psychosocial and behavioral risks with adverse pregnancy outcomes among African-Americans in Washington, DC. Matern Child Health J. 2011;15(1):8595. doi: 10.1007/s10995-011-0856-z

13. Mcintyre SH, Newburn-Cook CV, O'Brien B, Demianczuk NN. Effect of older maternal age on the risk of spontaneous preterm labor: a population based study. Health Care Women Int. 2009;30(8):670-689. doi: 10.1080/07399330802596473

14. Keely A, Gunning M, Denison F. Maternal obesity in pregnancy: women's understanding of risks. Braz J Microbiol. 2011;19(6):364-69. doi: 10.12968/bjom.2011.19.6.364

15. Rich-Edwards JW, Fraser A, Lawlor DA, Catov JM. Pregnancy characteristics and women's future cardiovascular health: an underused opportunity to improve women's health? Epidemiol Rev. 2013;36(1):57-70. doi: 10.1093/epirev/mxt006

16. Otterloo LRV, Connelly CD. Maternal risk during pregnancy: a concept analysis. J Clin Nurs. 2016;25(18):2393-401. doi: 10.1111/jocn.13294

17. Pereira MU, Solé D. Deficiência de vitamina D na gravidez e o seu impacto sobre o feto, o recém-nascido e na infância. Rev Paul Pediatr. 2015;33(1):104-113. doi: 10.1016/j.rpped.2014.05.004.

18. Firoozi F, Lemière $C$, Ducharme FM, Beauchesne MF, Perreault $S$, Bérard $A$, et al. Effect of maternal moderate to severe asthma on perinatal outcomes. Respirat Medic. 2010;104(9):1278-87. doi: 10.1016/j.rmed.2010.03.010

19. Hung TH, Chen SF, Hsu JJ. Gestational weight gain and risks for adverse perinatal outcomes: A retrospective cohort study based on the 2009 Institute of Medicine guidelines. Taiw J Obst and Gyn. 2015;54(4):421-25. doi: 10.1016/j.tjog.2015.06.010

20. Borthen I. Obstetrical complications in women with epilepsy. Seizure (Lond.). 2015;28(1):32-34. doi: 10.1016/j.seizure.2015.02.018 
21. Farmen AH, Grundt J, Tomson T, Nakken KO, Nakling J, Mowinchel P, et al. Intrauterine growth retardation in foetuses of women with epilepsy. Seizure (Lond.). 2015;28:76-80. doi: 10.1016/j.seizure.2015.02.026

22. Sunitha T, Prasoona KR, Kumari TM, Srinadh B, Deepika, MLN, Aruna, R, et al. Risk factors for congenital anomalies in high risk pregnant women: a large study from South India. Egypt J Medic Human Gen. 2017;18(1):79-85. doi: 10.1016/j.ejmhg.2016.04.001

23. Persson M, Cnattingius S, Wikström AK, Johansson S. Maternal overweight and obesity and risk of pre-eclampsia in women with type1 diabetes or type 2 diabetes. Diabetolog. 2015;59(10):2099-105. doi: 10.1007/s00125-016-4035-z

24. Mochhoury L, Razine R, Kasouati JKM, Barkat A. Body mass index, gestational weight gain, and obstetric complications in Moroccan population. J Pregn. 2016;2013(1):1-6. doi: 10.1155/2013/379461.

25. Cho EH, Hur J, Lee KJ. Early gestational weight gain rate and adverse pregnancy outcomes in Korean women. PloS One. 2015;10(10):e0140376. doi: 10.1371/journal.pone.0140376.

26. Fitzpatrick KE, Tuffnell D, Kurinczuk JJ, Knight M. Pregnancy at very advanced maternal age: a UK population based cohort study. Int J Obstet Gy. 2016;124(7):1097-106. doi: 10.1111/1471-0528.14269

27. Tandu-Umba B, Mbangama MA, Kamongola KMB, Tchawou AGK, Kivuidi MP, Munene SK, et al. Pre-pregnancy high-risk factors at first antenatal visit: how predictive are these of pregnancy outcomes?. Int J Womens Health. 2014;6(1):1011-18. doi: 10.2147/IJWH.S69230

28. Castaño IB, Sanchez PH, Perez NA, Salvador JJG, Quesada AG, Hernández JAG, et al. Maternal obesity in early pregnancy and risk of adverse outcomes. PLoS One. 2013;8(11):e80410. doi: 10.1371/journal.pone.0080410

29. Harada T, Taniguchi F, Onishi K, Kurozawa Y, Hayashi K, Harada T. Obstetrical complications in women with endometriosis: a cohort study in Japan. PLoS One. 2016;11(12):e0168476. doi: 10.1371/journal.pone.0168476

30. Zhang Y, Wang H, Pan X, Teng W, Shan Z. Patients with subclinical hypothyroidism before 20 weeks of pregnancy have a higher risk of miscarriage: a systematic review and meta-analysis. PloS One. 2017;12(4):e0175708. doi: 10.1371/journal.pone.0175708

31. Masukume G, Khashan AS, Kenny LC, Baker PN, Nelson G, Scope Consortium. Risk factors and birth outcomes of anaemia in early pregnancy in a nulliparous cohort. PloS One. 2015;10(4):e0122729. doi: 10.1371/journal.pone.0122729

32. Wallace JM, Bhattacharya S, Horgan GW. Weight change across the start of three consecutive pregnancies and the risk of maternal morbidity and SGA birth at the second and third pregnancy. PloS One. 2017;12(6):e0179589. doi: 10.1371/journal.pone.0179589

33. Luz AG, Tiago DB, Silva JCGD, Amaral E. Morbidade materna grave em um hospital universitário de referência municipal em Campinas, Estado de São Paulo. Rev Bras Ginecol Obstet. 2008;30(6):281-6. doi: 10.1590/S0100-72032008000600003

34. Chaves EGS, Franciscon PDM, Nascentes GAN, Paschoini MC, Silva APD, Borges MDF. Estudo retrospectivo das implicações maternas, fetais e perinatais em mulheres portadoras de diabetes, em 20 anos de acompanhamento no Hospital Escola da Universidade Federal do Triângulo Mineiro. Arq Bras Endocrinol Metabol. 2010;54(7):620-629. doi: 10.1590/S0004-27302010000700006

35. Loureiro C, Cataño CR, Torricelli L, Monteiro JCS, Gomes-Sponholz FA. Aspectos sociodemográficos y obstétricos de la morbilidad materna grave. Cienc y Enferm. 2017;23(2):21-32. doi: 10.4067/S0717-95532017000200021

36. Vidal CEL, Carvaho MAB, Grimaldi IR, Reis MC, Baêta MCN, Garcia RB, et al. Morbidade materna grave na microrregião de Barbacema/MG. Cad Saúde Colet. 2016;24(2): 131-6. doi: 10.1590/1414-462X201600020181

37. Nascimento MID, Cunha ADA, Guimarães EV, Alvarez FS, Oliveira SRDSM, Bôas V, et al. Gestações complicadas por sífilis materna e óbito fetal. Rev Bras Ginecol Obstet. 2011;34(2):56-62. doi: 10.1590/S0100-72032012000200003

38. Nomura RMY, Paiva LV, Costa VN, Liao AW, Zugaib M. Influência do estado nutricional materno, ganho de peso e consumo energético sobre o crescimento fetal, em gestações de alto risco. Rev Bras Ginecol Obstet. 2012;34(3): 107-112. doi: 10.1590/S0100-72032012000300003

39. Tinedo MJ, Santander F, Alonso J, Herrera A, Colombo C, Díaz M. Muerte fetal: caracterización epidemiológica. Salus [Internet]. 2016 [cited 2018 Jun 28];20(2):37-43. Available from: http://www.scielo.org.ve/pdf/s/v20n2/art08.pdf

40. Santos MDLM, Hernández JJR, Avich NS. Estrés psicosocial laboral como factor de riesgo para las complicaciones de la gestación y el bajo peso al nacer. Rev Cuba Salud Pública [Internet]. 2013 [cited 2018 Jun 28];39(1):864-880. Available from: http://scielo.sld.cu/pdf/rcsp/v39s1/spu06513.pdf

41. Lima MDOP, Tsunechiro MA, Bonadio IC, Murata M. Depressive symptoms in pregnancy and associated factors: longitudinal study. Acta Paul Enferm. 2017;30(1):39-46. doi: 10.1590/1982-0194201700007

42. González JAS, Guerrero RP, Machado MG, Delgado MRC, Tápanes YM, González VC. Influencia de la obesidade pregestacional em el riesgo de preeclampsia/eclampsia. Rev Cubana Obstet Ginecol [Internet]. 2013 [cited 2018 Jun 28];39(1):3-11. Available from: http://scielo.sld.cu/ pdf/gin/v39n1/gin02113.pdf

43. Bravo AID, Maya JVL, Paredes FCM. Mortalidad Perinatal de la Población afiliada a una EPS de Pasto. Departamento de Nariño. 2007. Hacia Promoc Salud [Internet]. 2015 [cited 2018 Jun 28];15(1):92-109. Available from: http://www.scielo.org.co/pdf/hpsal/v15n1/v15n1a07.pdf

44. Gonçalves ZR, Monteiro DLM. Complicações maternas em gestantes com idade avançada. Feminina [Internet]. 2012 [cited 2018 Jun 28];40(5):275-9. Available from: http://files.bvs.br/upload/S/0100-7254/2012/v40n5/a3418.pdf

45. Donoso B, Oyarzún E. Embarazo de alto riesgo. Medwave. 2012;12(5):e5429. doi: 10.5867/medwave.2012.05.5429

46. Silva KDL, Fernandes FEM, Pessoa TL, Lima SIVC, Oliveira AG, Martins RR. Prevalence and profile of adverse drug reactions in high-risk pregnancy: a cohort study. BMC Pregnancy and Childbirth. 2019;19(1):199-205. doi:10.1186/s12884-019-2321-8 
47. Lima THB, Katz L, Kassar SB, Amorim MM. Neonatal near miss determinants at a maternity hospital for high-risk pregnancy in Northeastern Brazil: a prospective study. BMC Pregnancy Childbirth. 2018;18(1):401-10. doi:10.1186/s12884-019-2381-9

48. Zhu X, Niu H, Wang H, Li X, Qi T, Ding W, et al. High risk pregnancy associated perinatal morbidity and mortality: a second birth populationbased survey in Huai'an in 2015. BMC Pregnancy Childbirth. 2019;19(1):224-8. doi: 10.1186/s12884-019-2323-6

49. Queiroz IS, Bertolin DC, Werneck AL. Complications and pre-existing diseases in pregnant women with diabetes mellitus. Rev Enferm UFPE. 2019;13(5):1202-8. doi: 10.5205/1981-8963-v13i05a238773p1202-1207-2019

50. Guerra JVV, Alves VH, Valete COS, Rodrigues DP, Branco MBLR, Santos MV. Gestational diabetes and pre-natal assistance at high risk. Rev Enferm UFPE. 2019;13(2):449-54. doi: 10.5205/1981-8963-v13i02a235033p449-454-2019

51. Veloso FCS, Kassar LDML, Oliveira MJC, Lima THB, Bueno NB, Gurgel RQ, et al. Analysis of neonatal mortality risk factors in Brazil: a systematic review and meta-analysis of observational studies. J Pediatr. 2019;95(5):519-30. doi: 10.1016/j.jped.2018.12.014

52. Nunes FBB, Silva PC, Barbosa TLSM, Lopes MLH, Silva EL. Influence of maternal age in perinatal conditions in live births of São Luís, Maranhão. Rev Pesqui: Cuid Fundam. 2020;12(1):281-7. doi: 10.9789/2175-5361.rpcfo.v12.8618

53. Ministério da Saúde (BR). Departamento de Informática do SUS. Informações de saúde (TABNET) [Internet]. Estatísticas Vitais. Brasília: MS; 2018 [cited 2018 Dec 18]. Available from: http://www2.datasus.gov.br/DATASUS/index.php?area=02 